\section{P34 OUTCOMES AFTER USING VEDOLIZUMAB IN PAEDIATRIC INFLAMMATORY BOWEL DISEASE IN A TERTIARY CENTRE, OVER A 3 YEAR PERIOD}

Rajkumar Dhandayuthapani, Vaia Zouzo, Fiona Cameron, Manjula Nair, Marcus Auth, Sarang Tamhne, Stephen Allen, Elizabeth Renji, Jeng Cheng. Alder Hey Children's Hospital

\subsection{6/flgastro-2021-bspghan.43}

Background Vedolizumab has proven efficiency in adults but data in paediatric inflammatory bowel disease (pIBD) is limited. We present the outcome of treatment with vedolizumab in refractory pIBD cohort.

Study Design Retrospective and ongoing prospective review of all patients commenced on Vedolizumab following loss of response to anti-tumour necrosis factor [TNF] between Nov 2017 and Nov 2020.

Aims and Objectives The primary outcome was remission at Week 14 and last follow up (wPCDAI/PUCAI $<10)$ from commencing vedolizumab. The secondary outcomes were to review trend of biochemical makers, surgical interventions, and adverse effects.

Results 11 children received vedolizumab (6[54\%] males), mean age at time of diagnosis $12.45(8.34-15.48)$ with a median(IQR) time from diagnosis of 2.68(1.79-5.28)years; 6 [54\%] Crohn's disease [CD] and 5[46\%] Ulcerative colitis/IBD Unclassified [UC/IBDU] (table 1).

For CD; $5 / 6$ was treated previously with anti-TNF $[40 \%$ primary failure, $60 \%$ secondary failure], all had colonic disease, 3/6 upper GI involvement and 3/6 perianal disease. One child with Bruton's agammaglobulinemia was anti-TNF naïve when commenced on vedolizumab. All UC children were treated with anti-TNF [40\% primary failure, $60 \%$ secondary failure], $80 \%$ had pan-colitis.

Median age at time of commencing vedolizumab (V0) was 14.99(13.0-17.6). Baseline characteristics at V0; faecal calprotectin(FC) 2851(92-6000), Hb 114(96-146), ESR 22(4-90), albumin 39(27-46) and CRP 16.7(4-39.5). 4/11(36\%) required surgery, three of whom had colectomy. 8/11 remained on immunomodulators with vedolizumab. Transient raised transaminases and eczema was reported once and low mood with suboptimal response noted once. 6/11(54\%) were in remission 14 weeks from commencing vedolizumab (V14) and 4/11(36\%) were excluded. At last follow up from commencing vedolizumab (VF), median years 2.21(0.78-3.43), 3 remained in remission.

In $\mathrm{CD}$ cohort, one child had a defunctioning ileostomy and remained in steroid free remission (SFR) at V14 and VF (3.43 years) on vedolizumab monotherapy. One had colectomy (FC-3296 wPCDAI-60), steroid dependency compounded by methotrexate induced interstitial nephritis and vedolizumab was discontinued at VF (2.19 years). Two continue to have active disease at V14 after commencing vedolizumab. One had SFR at V14 and was transitioned at 2 years (FC-2585, wPCDAI-25) on vedolizumab. One with anti-TNF resistant disease, achieved clinical remission 9 months after starting vedolizumab (wPCDAI 2.5, FC 598) before being transitioned.

In UC cohort, two had vedolizumab primary non-response needing subtotal colectomy. One patient with PUCAI 5 at V14 needed regime intensification for low vedolizumab levels but had active disease (PUCAI-25, FC-366) when transitioned at VF (2.06 years). One patient, who achieved remission whilst on steroid at V14, remains in SFR at VF (0.58 years) on concomitant immunomodulation and optimal vedolizumab level at
Abstract P34 Table 1

\begin{tabular}{|c|c|c|c|c|c|}
\hline & & \multicolumn{2}{|c|}{ Crohn's Disease } & \multicolumn{2}{|c|}{ Ulcerative Colitis } \\
\hline \multicolumn{2}{|c|}{ Completion of vedolizumab induction } & \multicolumn{2}{|l|}{$4 / 6$} & \multicolumn{2}{|l|}{$4 / 5$} \\
\hline \multicolumn{2}{|c|}{ Median (IQR) follow-up years from $V_{0}$} & \multicolumn{2}{|c|}{$2.76(0.04-3.43)$} & \multicolumn{2}{|c|}{$2.06(0.57-3.28)$} \\
\hline Remission at 14 weeks & Total^ & \multicolumn{2}{|c|}{$4(100 \%)$} & \multicolumn{2}{|c|}{$2(66.6 \%)$} \\
\hline$C D(n=4), U C(n=3)$ & Steroid free* & \multicolumn{2}{|c|}{$2(50 \%)$} & \multicolumn{2}{|c|}{$1(33 \%)$} \\
\hline Remission at last follow up & Total & \multicolumn{2}{|c|}{$2(50 \%)$} & \multicolumn{2}{|c|}{$1(33 \%)$} \\
\hline$C D(n=4), U C(n=3)$ & Steroid free & \multicolumn{2}{|c|}{$2(50 \%)$} & \multicolumn{2}{|c|}{$1(33 \%)$} \\
\hline \multirow[t]{3}{*}{ PUCAI/PCDAI median (range) } & $\mathrm{V}_{0}$ & 37.5 & $(0-67.5)$ & 40 & $(30-60)$ \\
\hline & $\mathrm{V}_{14}$ & 6.25 & $(5-10)$ & 5 & $(0-15)$ \\
\hline & $V_{F}$ & 25 & $(0-60)$ & 22.5 & $(15-25)$ \\
\hline \multirow[t]{3}{*}{ Calprotectin median (range) } & $\mathrm{V}_{0}$ & 2214.5 & $(92-6000)$ & 3702 & $(143-5075)$ \\
\hline & $\mathrm{V}_{14}$ & 185 & $(30-4228)$ & 126 & (8-244) \\
\hline & $V_{F}$ & 2332 & $(31-6000)$ & 862.5 & $(366-6000)$ \\
\hline \multirow[t]{2}{*}{ Weight centile median (range) } & $\mathrm{V}_{0}$ & 30 & $(3-75)$ & 42 & $(31-99)$ \\
\hline & $V_{F}$ & 41 & $(29-84)$ & 48.5 & (54-99) \\
\hline
\end{tabular}

$\wedge$ Total number of patients in remission, with or without concomitant steroid treatment

*Total number of patients in remission, without concomitant steroid treatment

$V_{14}-14$ weeks from time of commencing vedolizumab

$\mathrm{V}_{\mathrm{F}}$ - point of last follow up from starting vedolizumab

end of induction $(>19)$. One who was lost to follow-up during COVID, was transitioned on 4 weekly vedolizumab regime.

Conclusion At V14, 54\% of patients achieved clinical remission and we see significant improvement with PUCAI/PCDAI scores and faecal calprotectin in both UC and CD cohort. We are continuing this study over a longer period to achieve a larger cohort.

\section{P35 OUTCOMES IN CHILDREN WITH PRIMARY SCLEROSING CHOLANGITIS OR AUTOIMMUNE HEPATITIS-OVERLAP AND ASSOCIATED INFLAMMATORY BOWEL DISEASE}

Kavitha Jayaprakash, Ellen Paling, Olivia Bradshaw, Marumbo Mtegha. Leeds General Infirmary

\subsection{6/flgastro-2021-bspghan.44}

Background Primary Sclerosing Cholangitis (PSC) and Autoimmune liver disease (autoimmune hepatitis \& overlap syndrome $(\mathrm{AIHO}))$ are rare entities. PSC and AIHO have been reported to be associated with IBD.

Aim To study outcomes of children diagnosed with AIHO and PSC who also have a diagnosis of inflammatory bowel disease (IBD). Outcomes included portal hypertension, biochemical remission, survival of native liver and mortality.

Methods This is a retrospective study (2000-2020) of 193 patients diagnosed with AIH. Of these, 23 patients (14M:9F) had diagnosis of either AIHO+IBD or PSC+IBD. None had $\mathrm{AIH}$ without overlap. Case notes were examined at intervals of $1,3,5,7$ and 10 years. Data included treatment modality, biochemical remission (ALT $<50 \mathrm{iu} / \mathrm{l})$, GGT $(<40 \mathrm{iu} / \mathrm{l})$, development of portal hypertension (platelet count $<120$, splenomegaly and varices) and survival of native liver.

Results 23 (12\%) patients were identified, of which 14 (60\%) had a diagnosis of PSC+ IBD (Crohn's: 6, indeterminate: 7, UC:1). Of the PSC group, 35\% had gastric, duodenal and colonic disease with 1 patient undergoing pan-proctocolectomy. All but one were diagnosed with IBD prior to PSC or concomitantly. The remaining $40 \%$ patients had AIHO+IBD 


\begin{tabular}{|c|c|c|c|c|c|c|}
\hline \multicolumn{7}{|c|}{ Primary Sclerosing Cholangitis \& IBD $-n=14$} \\
\hline Order of diagnosis & \multicolumn{6}{|c|}{13 diagnosed with IBD prior/concomitant to PSC } \\
\hline Age @ Diagnosis & \multicolumn{6}{|c|}{ Median - 9 years (5-16) } \\
\hline \multirow[t]{2}{*}{ Type of IBD } & $C D$ & IBDU & UC & & & \\
\hline & 6 & 7 & 1 & & & \\
\hline Location & \multicolumn{2}{|c|}{ Gastric, duodenal, colonic } & colonic & & & \\
\hline \multirow[t]{2}{*}{ Treatment } & 5-ASA & CS & Aza & Biologics & UDCA & Surgical \\
\hline & $57 \%$ & $21 \%$ & $14 \%$ & $15 \%$ & $64 \%$ & $1(? \%)$ \\
\hline \multirow[t]{2}{*}{ GGT (median) } & diagnosis & $1 \mathrm{yr}$ & $7 \mathrm{yr}$ & & & \\
\hline & 268 & 37 & 31 & & & \\
\hline
\end{tabular}

\begin{tabular}{|c|c|c|c|c|c|c|}
\hline \multicolumn{7}{|l|}{ AlHO \& IBD - n = 9} \\
\hline \multirow[t]{2}{*}{ Order of diagnosis } & \multicolumn{6}{|c|}{ AlH diagnosed prior to IBD - 4} \\
\hline & \multicolumn{6}{|c|}{ IBD diagnosed prior to $\mathrm{AIH}-3$} \\
\hline Age at Diagnosis & \multicolumn{6}{|c|}{ Median - 12 years (6-16) } \\
\hline \multirow[t]{2}{*}{ Type of IBD } & IBDU & & UC & & & \\
\hline & 2 & & 7 & & & \\
\hline Location & \multicolumn{6}{|l|}{ Colonic } \\
\hline \multirow[t]{2}{*}{ Treatment } & 5-ASA & CS & Aza & Surgical & UDCA & Biologics \\
\hline & $22 \%$ & $100 \%$ & $44 \%$ & $33 \%$ & $55 \%$ & $4 \%$ \\
\hline
\end{tabular}

(UC:7, indeterminate: 2). AIHO was diagnosed prior to IBD in $4 ; 3$ were diagnosed with IBD prior to $\mathrm{AIH}$. In this group, $66 \%$ had severe pancolitis and 33\% required surgical resection. (Results summarised in table below).

In the PSC+IBD group follow-up data included 1 year $(\mathrm{n}=8), 3$ year $(\mathrm{n}=6), 5$ year $(\mathrm{n}=5) 7$ year $(\mathrm{n}=3)$. Median age at diagnoses was 9 years (5-16). Aside from one patient, patients were diagnosed with IBD prior to PSC or concomitantly. At diagnosis, 64\% received ursodeoxycholic acid; $21 \%$ had steroids, $14 \%$ had azathioprine and $57 \%$ had aminosalicylates. Two patients received infliximab and adalimumab. The median Gamma GT (GGT) at diagnosis was 268 (16-877), at 1 year was 37 (7-104), at 7 years was 31 (11-52).

In the AIHO $+\mathrm{IBD}$ group, data included 1 year $(\mathrm{n}=8), 3$ year $(n=3), 5$ year $(n=1)$ and 7 year $(n=1)$. Median age at diagnoses was 12 years (6-16). AIH was diagnosed prior to IBD in 4 patients; 3 patients were diagnosed with IBD prior to $\mathrm{AIH}$. At diagnosis, $55 \%$ received ursodeoxycholic acid, $100 \%$ received steroids, 44\% received azathioprine and 22\% of patients received aminosalicylates. One year after diagnosis, one patient received infliximab. Biochemical remission was achieved in $75 \%$ (5) by 1 year and 100\% (3) by three years.

In both groups, normalisation of GGT or ALT did not correlate with resolution of endothelial thickening of the $\mathrm{CBD}$ on ultrasound. Portal hypertension was found in 13\% of patients (PSC group 2; AIHO 1). No patients died or received a liver transplant.

Summary and Conclusion Patients with PSC were more likely to have a diagnosis of IBD-U whilst patients with AIH-overlap were more often diagnosed with UC. In terms of IBD outcomes $13 \%$ of patients required biologics and surgical resection. In keeping with the literature, only one patient had portal hypertension at diagnosis. In total, $13 \%$ of $\mathrm{AIHO}+\mathrm{IBD}$ and PSC+IBD patients developed portal hypertension. None died or required liver transplantation.

\section{P36 OUTCOMES OF PEDIATRIC LIVER TRANSPLANTATION, COMPARISON BETWEEN ACUTE AND CHRONIC LIVER FAILURE SETTINGS}

${ }^{1}$ Amr Alnagar, ${ }^{2}$ Khaled Daradka, ${ }^{2}$ Eirini Kyrana, ${ }^{2}$ Marumbo Methga, ${ }^{2}$ Karthikeyan Palaniswamy, ${ }^{2}$ Suzanne Davison, ${ }^{2}$ Patricia Mcclean, ${ }^{2}$ Sanjay Rajwal, ${ }^{2}$ Vivek Upasani, ${ }^{2}$ Vijayanand Dhakshinamoorthy, ${ }^{2}$ Raj Prasad, ${ }^{2}$ Magdy Attia. ${ }^{1}$ Birmingham Women's and Children's NHS Foundation Trust; ${ }^{2}$ Leeds Teaching Hospitals NHS Trust

\subsection{6/flgastro-2021-bspghan.45}

Background Managing children suffering from acute liver failure (ALF) is a dynamic process. Listing them for liver transplantation (LT) is considered when the probability of spontaneous recovery is low. Children with ALF who meet criteria are eligible for super urgent transplantation, the window between presentation and LT can range between few hours up to few days. The dynamics are different in case of children with end stage chronic liver disease (ESCLD) who are transplanted electively as candidates are usually in less critical condition.

Aim To compare long-term recipient and graft survival as well as complication rates between children transplanted for either ALF or ESCLD.

Methods This is a retrospective review of primary LT recipients in Leeds Teaching Hospitals NHS trust. Patients were divided into either ALF or ESCLD group according to their listing indication and the following parameters were compared: Pre-transplant recipient parameters, donor parameters, operative parameters, and outcomes.

Results Children undergoing re-transplantation, transplants for liver tumours or metabolic diseases without underlying

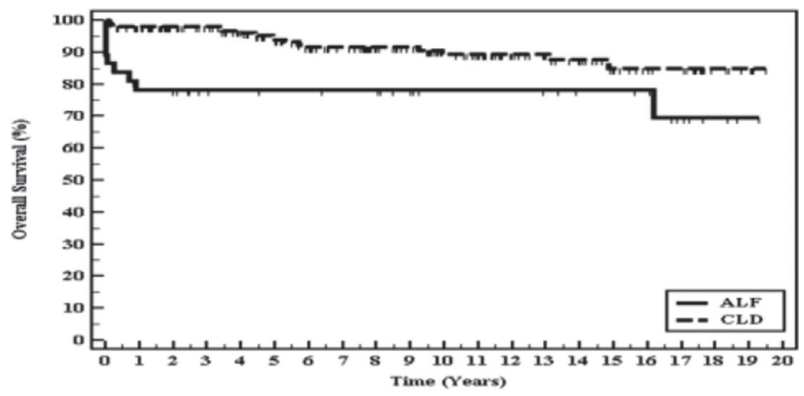

Abstract P36 Figure 1 Recipient survival curves for both groups Figure2: graft survival curves for both groups

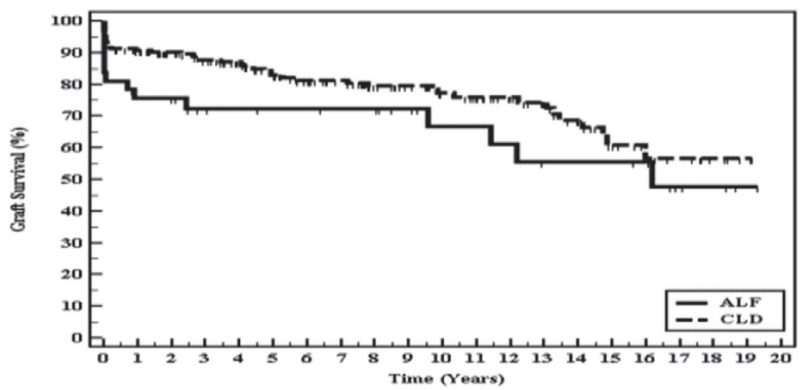

Abstract P36 Figure 2 Graft survival curves for both groups 